X.

Aus der II. inneren Abteilung und dem hydrotherapeutischen Institut des Rudolf Virchow-Krankenhauses zu Berlin.

\title{
Ueber die Aenderungen des Elektrocardiogramms von Herzkranken durch Kohlensäurebäder.
}

\author{
Von
}

\section{Prof. Dr. Kurt Brandenburg und Dr. August Laqueur.}

(Mit 3 Curren im Text.)

An Kranken mit Zuständen von Herzmuskelschwäche verschiedener Ursache und Stärke, sowie an Kranken mit vorwiegend auf nervöser Grundlage entwickelten Herzbeschwerden wurden Untersuchungen über die Beeinflussung des Elektrocardiogramms durch Kohlensäurebäder angestellt. Die Befunde wurden in Beziehung gesetzt $\mathrm{zu}$ dem klinischen Gesamtbilde und zu anderen gleichzeitig geprüften Functionen, wie Blutdruck, Zwerchfellstand und galvanischem Leitungswiderstand des Körpers.

Dabei wurden Erfahrungen gewonnen, die von einem gewissen praktischklinischen und cardiographischen Interesse sind. Im folgenden sollen einige dieser Ergebnisse berichtet und an der Hand von Beispielen erläutert werden.

Untersuchungen über den Einfluss von Badeproceduren auf die Form des Elektrocardiogramms sind bereits von verschiedenen Seiten mitgeteilt worden. Elektrocardiogramme auch nach Anwendung von kühlen Kohlensäurebädern sind am Menschen und im Tierversuch aufgezeichnet worden. In Kürze sei darauf Bezug genommen, soweit die hier berührten Fragen dabei behandelt worden sind.

Der Einfluss von Bäderproceduren auf das Elektrocardiogramm wurde im Zusammenhang wohl zuerst auf dem Berliner Balneologencongress 1912 besprochen, und zwar yon 3 Referenten Nicolai, Bickel und Strubell.

Nicolai (1) berichtete zunächst über die Ergebnisse der Arbeit von Reinboldt und Goldbaum, auf die später noch näher eingegangen werden soll, und ergänzte deren Resultate durch die Mitteilung von Untersuchungen seines Schülers Golodecz über die Beeinflussung des Elektrocardiogramms durch Solbäder ( 6 proc.) von $34^{0}$. Es ergab sich dabei, dass bei den berzgesunden Versuchspersonen constant eine erhebliche Erhöhung der F-Zacke durch diese Bäder erzielt wurde, daneben eine starke Verkleinerung der Ip-Zacke. Bөi gewöhnlichen Bädern ohne Zusatz waren diese Veränderungen viel weniger ausgesprochen und auch weniger constant. Zusammenfassend schliesst Nicolai aus den vorliegenden Untersuchungen, dass durch Bäder die einzelnen Zacken des Elektrocardiogramms beeinflusst werden, am deutlichsten die F-Zacke. Diese wird sowohl durch warme wie kalte Bäder erh öht, durch letztere jedoch mehr, wenn auch nicht so anhaltend wie durch warme Bäder. Kalte Bäder zeigen die Tendenz, die I-Zacken zu verkleinern, warme, sie zu erhöhen. Die Vorhofzacke verhält sich inconstant, hat aber bei beiden Bäderarten die Tendenz zur Vergrösserung. Durch Hinzufügung von gebundenen $\mathrm{Gasen}\left(\mathrm{CO}_{2}\right.$ und $\left.\mathrm{O}_{2}\right)$ so wie von Sole wird die Wirkang der kalten Applicationen auf das Herz verstärkt resp. ersetzt.

Nicolai erwähnt, dass die vorliegenden Resultate durch Untersuchungen an Herzkranken ergänzt werden müssten. Ein Anfang dazu ist schon in der Arbeit 
von Reinboldt und Goldbaum (2) gemacht. Dieselben stellten spociell ihre Untersuchungen über die Wirkung der $\mathrm{CO}_{2}$-Bäder sowohl an Patienten mit normalem Herzbefund resp. mit völlig compensierten Herzaffectionen als auch an Kranken mit leicht gestörter Kompensation an. Ein Unterschied in der Wirkung der $\mathrm{CO}_{2}$-Bäder auf das Elektrocardiogramm - Erhöhung der I- und F-Zacke nach dem Bade nach anfänglicher Verkleinerung, ohne dass dabei meist die ursprüngliche Zackenhöhe überschritten wurde - liess sich bei diesen beiden Gruppen von Versuchspersonen nicht feststellen; nur in einem Falle von Compensationsstörung (Versuch 14) war die Erhöhung derZacken erheblicher als bei den functionell herzgesunden Patienten. Im ganzen waren die Resultate nicht immer constant, jodoch waren im Vergleich zu anderen Bädern die Schwankungen der Zackenhöhe nach $\mathrm{CO}_{2}$-Bädern am stärksten und gesetzmässigsten.

DieVersuche Bickels (3) und seinerMitarbeiter[Rhein boldt (4), Waledinsky (5)] wurden an Kaninchen ausgeführt und erstreckten sich auf einfach $\theta$ indifferente $\left(36-37^{\circ}\right)$ Wasserbäder und ebensolche $\mathrm{CO}_{2}$ - und 0 -Bäder. Nach einfachen Wasserbädern fanden diese Autoren eine Erniedrigung aller Zacken, namentlich der J-Zacke. Nach Sauerstoffbädern ist diese Abnabme nicht continuierlich, namentlich die F-Zacke verhält sich hier der erniedrigenden Wirkung des indifferenten Süsswasserbades gegenüber resistent (Rheinboldt). Im $\mathrm{CO}_{2}-\mathrm{Bade}$ werden alle Zacken erniedrigt, und zwar mebrals im einfachen Wasserbade, vor allem wieder die l-Zacke, weniger die A-Zacke und die noch resistentereF-Zacke. Bemerkenswert ist, dass bei Wiederholung der Procedur bei demselben Tier die Beeinflussung der Zackenhöhe durch das $\mathrm{CO}_{2}$-Bad eine geringer $\theta$ wird (Waledinsky). Dass die Veränderung der Zackenhöhe nicht etwa durch Einatmung des $\mathrm{CO}_{2}$-Gases bedingt wird, zeigten Versuche an Tieren mit künstlicher Respiration, bei denen sich dieselben Wirkungen der $\mathrm{CO}_{2}$-Bäder ergaben.

$\mathrm{Zu}$ erwähnen sind hier ferner die Untersuchungen Strubells (6) über die Beeinflussung des Elektrocardiogramms durch Wechselstrombäder, die ja manche Analogie mit den $\mathrm{CO}_{2}$-Bädern bezüglich ihrer Circulationswirkungen zeigen. Strubell fand bei Herzkranken durchweg eine Erhöhung derF-Zacke nach einer Kur mit Wechselstrombädern; nur in Fällen von thyreotoxischer Herzvergiftung, wo an sich schon die F-Zacke eine pathologische Erhöhung zeigt, wurde durch die Wechselstrombäder diese Zacke in umgekehrtem Sinne beeinflusst, also erniedrigt. Durch die nach derStrubellschen Technik applicierten Bäder wurde übrigens der Blutdruck in der Mohrzahl der Fälle orniedrigt.

Ein ausdrückliches Eingehen auf die Beziehungen von Blutdruck und Elektrocardiogramm unter dem Einfluss von Bädern finden wir bei Jastrowitz (7). Er fand, dass nach heissen Proceduren eine Zunahme der Zackenhöhe, nach kalten im allgemeinen eine Abnahme derselben stattfindet. Doch zeigte sich nach energischen Kälteproceduren, dass gleichzeitig mit dem Absinken der Höhe von I und $\mathrm{F}$ auch ein Absinken des Ventrikelquotienten $I: F$, also ein relatives Anwachsen von $F$ bei gleichzeitiger Erhöhung des Blutdrucks erfolgt. Mit $\mathrm{CO}_{2}$-Bädern hat Jastrowitz im ganzen 4 Versuche angestellt (Bädertemperatur $32-33^{0}$, Daver 15-20 Min.) Es fand sich dabei einmal eine Zunahme, sonst aber eine Abnahme der Höhe der F-Zacke, 3 mal geringe Zunahme, einmal Abnahme der F-Zacke, 3 mal mehr minder gering $\theta \mathrm{Abnahme}$ des Quotienten $\frac{\mathrm{l}}{\mathrm{F}}$ bei gleichzeitiger vorübergehender Blutdruckerhöhung. Alle Untersuchungen Jastrowitz' sind an 2 herzgesunden Personen ausgeführt worden.

Schliesslich sei noch als klinisoh wichtig eine Mitteilung Theo Grödels (8) erwäbnt, der in einem Fall von Herzaffection mit atrioventriculärem Block nach einer Kohlensäurebäder-Kur nicht nur klinische Besserung, Beseitigung der Oedeme, Erböhung der Pulsfrequenz usw., sondern auch im Elektrocardiogramm eine zeitweilige Wiederherstellung der normalen Reizleitung beobachten konnte, während Medikamente oinen derartigen Einfluss nicht ausübten. 
Aus den Angaben der Bearbeiter sei hervorgehoben: Bei gesunden Versuchspersonen scheint eine bestimmte Reaction feststellbar zu sein. Kühle Bäder erniedrigen die Initialzacke des Kammerelektrocardiogramms, dagegen nicht die Finalschwankung, die zuweilen vergrössert wird. Die Folge der verschiedenen Beeinflussung ist die Aenderung des Quotienten aus beiden, I/F, im Sinne einer Verkleinerung. Gleichzeitig steigt der Blutdruck. Dic untersuchten Herzkranken zeigten keine wesentlichen Unterschiede gegenüber den Herzgesunden.

Die Aufgabe der nachstehenden Untersuchungen war: Zunächst dieAenderungen festzustellen, die bei Kranken mit Herzbeschwerden verschiedener Art und Entstehung am Elektrocardiogramm auftreten. Weiter galt es, diese Aenderungen zu deuten und aus den Curven Zusammenhänge mit bekannten physikalischen Veränderungen und mit dem klinischen Zustande abzuleiten.

\section{Die Untersuchungsmethodik.}

Die Untersuchungen wurden unter gleichmässigen Bedinguugen vorgenommen. Die längere Zeit beobachteten Kranken wurden etwa fünf Minuten vor und spätestens zehn Minuten nach dem Kohlensäurebad untersucht. Es ist wichtig, dass die Kranken in gleicher Weise vorbereitet sind. Für die Beurteilung der Bäderwirkung ist es nicht gleichgültig, ob der Kranke eine halbe Stunde im Garten gegangen ist oder ob er ausgeruht und aus dem Bett zur Untersuchung und zum Bade kommt.

Bestimmt wurden vorher und nachher Blutdruck, nach der auscultatorischen Methode (Korotk off-Fellner), Pulsfrequenz und Stand der unteren Lungengrenzen. Im Laufe der Untersuchungen stellte sich die Notwendigkeit heraus, gleichzeitig mit dem Elektrocardiogramm auch den Widerstand im Ableitungskreise gegen einen constanten Strom bekannter Voltspannung zu bestimmen.

Gearbeitet wurde mit dem Elektrocardiographen von Siemens \& Halske. Das brauchbare Instrument hat Vorzüge vor dem Saitengalvanometer. Die Messschleife ist in ihrer Empfindlichkeit bequem einstellbar und ihre Ausschläge geben das genaue Bild der Stromschwankungen. Der Gewinn, der aus der Einführung der Schleife an Stelle der Saite entspringt, ist ein sehr bedeutender. Denn alle die Nachteile fallen fort und damit auch die Schwierigkeiten, die sich aus der ungenügend, zu stark oder zu schwach gespannten Saite ergeben und der dadurch bedingten fehlerhaften Schreibung der Herzcurve. Bei dem Messschleifensystem gelingt es auch, im Gegensatz zur Saite, in jedem Falle leicht, die Aperiodicität der Schwingungen mit zureichender Anspruchsfähigkeit des Systems in Einklang zu bringen. Beides erfolgt unabhängig von einander, die Einstellung auf Empfindlichkeit durch die Ausschaltung eines zur Schleife parallel geschalteten Widerstandes, und die Einstellung auf Aperiodicität durch Regulierung des Accumulatorenstromes für den Magneten. Dadurch ist der Ausschlag des Systems aut Aperiodicität und Stromempfindlichkeit jederzeit unschwer der Prüfung und Aenderung zugänglich. Es ist also die Schleife in der Ausführung und Anordnung des Siemens \& Halskeschen Instrumentes ein vortrefflich verwendbares und zuverlässiges Ampèremeter und ein für die klinische Praxis sehr brauchbarer Elektrocardiograph. 
Durch Eichung mit einem Strom bekannter Spannung ist der Ampèrewert der Ausschläge am Instrument bestimmbar und durch Bestimmung der Widerstände im jedesmaligen Ableitungskreise gegen eine Spannung von 1,5 Volt des constanten Stromes, gleichfalls am Instrument, auch das Verhältniss ihrer Voltwerte. Es wurde mit der für diese Zwecke ausreichenden Condensatoreinschaltung in den Stromkreis gearbeitet. Beim liegenden Kranken wurden jedesmal zwei Aufnahmen nach einander gemacht, mit Ableitung I von beiden Armen und mit Ableitung von den auf die Brust-haut - Herzspitze und rechter Armschlüsselbeinwinkel - aufgesetzten unpolarisierbaren Trichterelektroden, bei denen ein Zinkstab in Zinksulfatlösung eintaucht, die durch tierische Membran abgeschlossen ist. Die kreisförmige Ableitungsfläche hatte $12 \mathrm{~cm}$ Durchmesser und drückte mit etwa $500 \mathrm{~g}$ Belastung. Die Ableitung von der Brust gab die Möglichkeit, auch bei unruhigen Herzkranken mit zitternden Händen nicht verzitterte und ausmessbare Curven zu gewinnen. Die Curven beider Ableitungen sind nicht unter einander vergleichbar und die Werte ihrer Erhebungen sind ganz verschieden.

\section{Das Elektrocardiogramm vor und nach dem Kohlensäurebade.}

An der Hand von Beispielen sollen zunächst einige Reactionsformen bei Herzkranken geschildert werden. Dabei ergeben sich die Beziehungen zwischen dem klinischen Bilde und dem Elektrocardiogramm.

1. Ju., Arbeiter, 64 Jahre. Mässiges Lungenemphysem, Arteriosklerose, Aorta rechts bis zum Sternoclaviculargelenk, Herz beiderseits mässig verbreitet. Bei Arbeit Fussödem und Herzbeschwerden. Die Bäder werden gut vertragen (Curve la u, lb).

Curven 1 a und $1 \mathrm{~b}$. Pat. Ju.

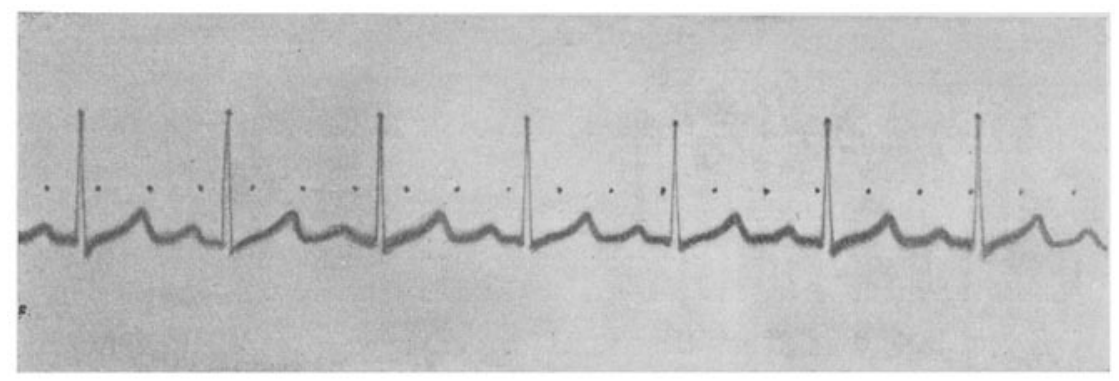

Vor dem Bade.

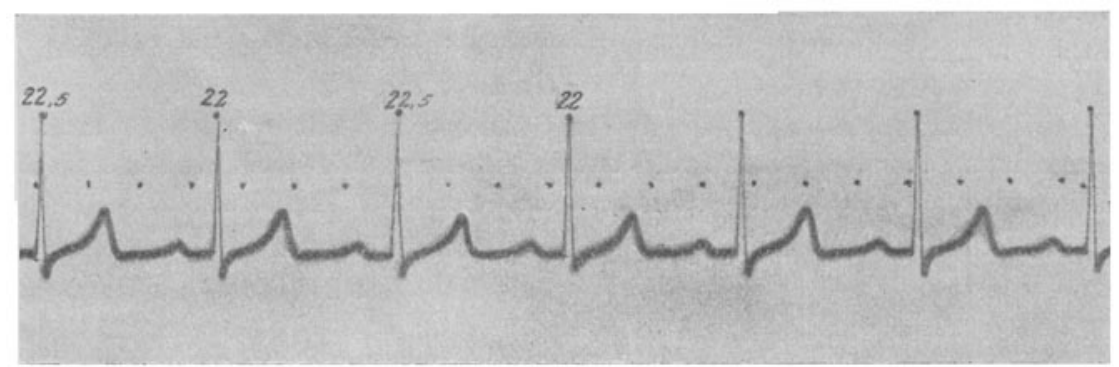

8 Minuten nach dem ersten Kohlensäurebade von $33^{\circ} ; 15$ Minuten Dauer.

In beiden Curven: Gesehwindigkeit $40 \mathrm{~mm}$ in der Seeunde; Zeitschreibung 0,2 Secunden. Empfindlichkeit des Systems: $1 \mathrm{~mm}$ Aussehlag $=0,9 \cdot 10^{-8} \mathrm{~A}$. 
31. 10. 1913. 1. Bad. Temperatur 33॰. Dauer 15 Minuten.

\begin{tabular}{|c|c|c|c|c|c|c|}
\hline & & Puls & Blutdruck & $\mathrm{I}(\mathrm{mm})$ & $\mathrm{F}(\mathrm{mm})$ & $\mathrm{I} / \mathrm{F}$ \\
\hline .-Elektr. v & & 92 & $115-174$ & 19,7 & 4,5 & 4,3 \\
\hline nach " & & 72 & $128-190$ & 21,9 & 6,8 & 3,2 \\
\hline
\end{tabular}

Untere Lungengrenzen durch Bad nicht verschoben.

Ergebnis: J. fühlt sich durch das Bad erfrischt. 10 Minuten nach dem Bade: Lungengrenzen nicht verschoben, Puls verlangsamt, Blutdruck erhöht. Elektrocardiogramm: Beide Kammerzacken vergrössert, aber die Finalschwankung im Verhältnis zur Initialschwankung mehr, so dass der Quotient I/F erniedrigt ist.

2. Po., Arbeiter, 50 Jahre. Alte Aorten- und Mitral-Insufficienz nach Gelenkrheumatismus in der Jugend. Wassermannsebe Reaction 0. Seit einem balben Jahre erste Erscheinungen ron Insufficienz des Herzens. Bei Aufnahme am 12. 8. 1913 starke Oedeme und Cyanose, durch Digitalis beseitigt. P. bleibt bei intermittierender Digitalisierung compensiert mit Pulsus irregularis perpetuus mit normalen Kammercomplexen.

6. 11. 1913. 1. Bad. 'Temperatur $33^{\circ}$. Dauer 15 Minuten.

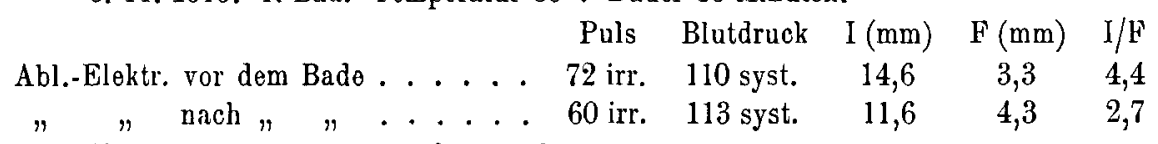

Untere Lungengrenzen nicht verschoben.

Ergebnis: Bad insofern schlecht vertragen, als vorübergehendes Beklemmungsgefühl und Unbehagen im Bade auftreten. 10 Minuten nach dem Bade: Lungengrenzen nicht verschoben, Blutdruck wenig erhöht. Elektrocardiogramm: Initialzacke niedriger, Finalschwankung grösser als vor dem Bade, also der Quotient $\mathrm{I} / \mathrm{F}$ bedeutend verkleinert.

Wegen dieser objectiv nachweisbaren günstigen Reaction werden trotz der anfänglichen Beschwerden im Wasser die Bäder fortgesetzt und vom 3. Bade an auch gut vertragen.

17. 11. 1913. 5. Bad. Temperatur $32,5^{\circ}$. Dauer 15 Min.

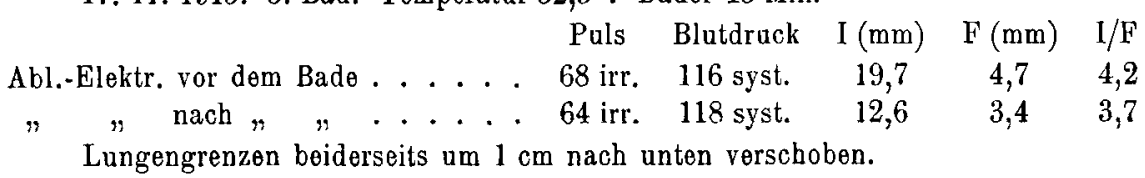

Ergebnis: Bad ohne Beschwerden vertragen. 10 Minuten nachher: gleichsinnige Aenderungen wie nach dem 1. Bade. Blutdruck wenig erhöht. Elektrocardiogramm: Initialzacke stark und Finalschwankung wenig verkleinert. - Die weiteren Bäder werden gut vertragen, auf die anfänglich durchgeführte schwache Digitalisierung wird im weiteren Verlauf verzichtet.

3. Och., Arbeiter, 19 Jahre. Vor 6 Wochen mit seiner ersten Polyarthritis rheumatica und Endocarditis mitralis auf die Abteilung aufgenommen, seit 3 Wochen beschwerdetrei. Frische Mitralinsufficienz ohne Compensationsstörungen.

3. 10. 1913. 4. Bad. Temperatur $32^{\circ}$. Dauer 15 Minuten.

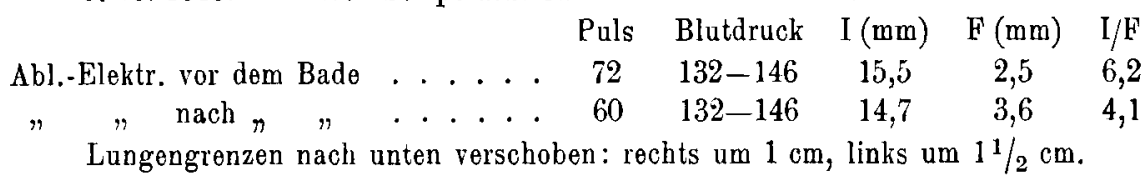


Ergebnis: Bäder ohne Beschwerde vertragen. 10 Minuten nach dem 4. Bade: Puls wird verlangsamt und Blutdruck unverändert gefunden. Untere Lungengrenzen nach unten verschoben. Elektrocardiogramm: Initialzacke niedriger, Finalschwankung höher als vor dem Bade, also Ventrikelquotient erheblich verkleinert.

In diesen geschilderten Fällen wurden die Kohlensäurebäder gut vertragen bis auf das 1. Bad bei Patient P., das Beklemmungen auslöste. Aber gemeinsam ist sämtlichen Fällen der Befund einer kräftigen und noch 10 Minuten nach dem Bade nachweisbaren Reaction. Der Eintritt der inspiratorischen Stellung des Zwerchfells ist abhängig von der Ausdehnungsfähigkeit der Lunge, er blieb aus in dem Falle von Lungenemphysem, er wurde aber auch vermisst nach dem ersten Beklemmungen verursachenden Bade bei P., wo der normale Reflex auf das Atemcentrum anscheinend ausgeblieben war. Regelmässig war der Puls verlangsamt. Die Steigerung des Blutdrucks ist bei dem jugendlichen Kranken Och. 10 Minuten nach dem Bade nicht nachweisbar; es ist anzunehmen, dass sie bereits abgeklungen war und in diesem Falle nicht so lange dauerte wie die Pulsverlangsamung und die Aenderung des Elektrocardiogramms. Dieses zeigt in allen angeführten Beispielen eine Vergrösserung der Finalschwankung gegenüber der Initialzacke, also ein Kleinerwerden des Quotienten I/F. Dabei werden die beiden Erhebungen gegenüber den Werten vor dem Bade bald grösser und bald kleiner geschrieben.

Diesen Fällen sei eine zweite Reihe von Beobachtungen gegenübergestellt.

4. Re., Handlungsgehilfe, 20 Jahre. Vor 6 Wochen die erste Polyarthritis rheumatica im Krankenhause überstanden. In deren Verlauf Endocarditis und Mitralinsufficienz. Jetzt keine Beschwerden, jedoch starke vasomotorische Erregbarkeit und Zittern der geschlossenen Lider und gestreckten Finger.

10. 10. 1913. 5. Bad. Temperatur $31,5^{0}$. Dauer 15 Minuten.

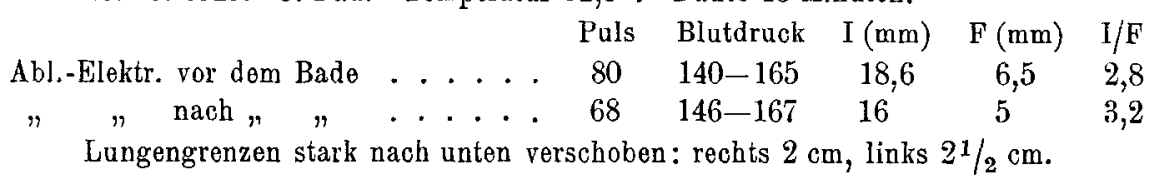

Ergebnis: Bad gut vertragen. 10 Minuten nach dem Bade: Puls verlangsamt, Blutdruck wenig erhöht, inspiratorische Dauerstellung. Elektrocardiogramm: Beide Erhebungen niedriger als vorher, die Finalschwankung mehr als die Initialzacke; der Ventrikelquotient ist also in diesem Falle grösser geworden als er vor dem Bade war.

Es ist von Interesse, diesen Befund zu vergleichen mit der Reaction desselben Kranken auf ein gewöhnliches kühles Vollbad wenige Tage später.

14. 10. 1913. Einfaches Vollbad. Temperatur 33-32 ${ }^{\circ}$. Dauer 15 Minuten.

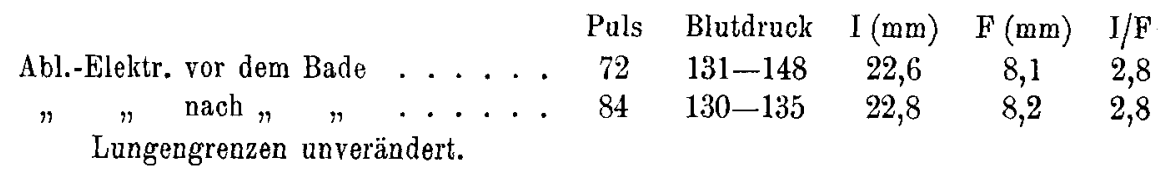


Ergebnis: 10 Minuten nach dem Vollbad ist eine Beeinflussung nicht festzustellen. Lungengrenzen und Blutdruck unverändert, und auch das Elektrocardiogramm ist vorher und nachher gleich geblieben.

Bei dem für nervöse Reize des Gefässsystems sehr zugänglichen Kranken war im Gegensatze zum Kohlensäurebade 10 Minuten nach einem einfachen kühlen Bade eine Reaction mit keinem der verwendeten Prüfungsmittel, auch nicht mit Hilfe des Elektrocardiogramms, nachweisbar.

5. Me., Bierfahrer, 45 Jabre. Invalide. Lebercirrhose ohne Stauungen, Arteriosklerose, systolisches Mitralgeräusch, Depressionszustände; Wassermannsche Reaction 0; Lid- und Fingertremor. M. leidet unter einer zeitweise verstärkt auftretenden extrasystolischen Aryhthmie (Curve 2a und 2b).

Currven $2 \mathrm{a}$ und $2 \mathrm{~b}$. Pat. Me.

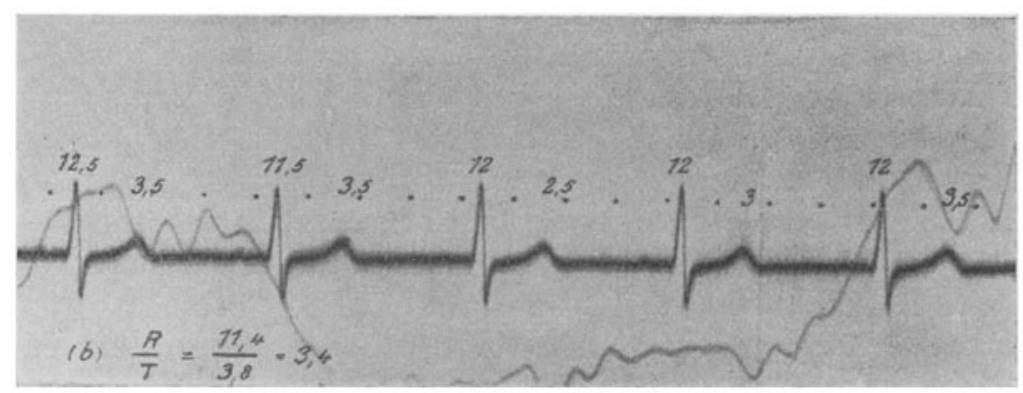

Vor dem Bade.

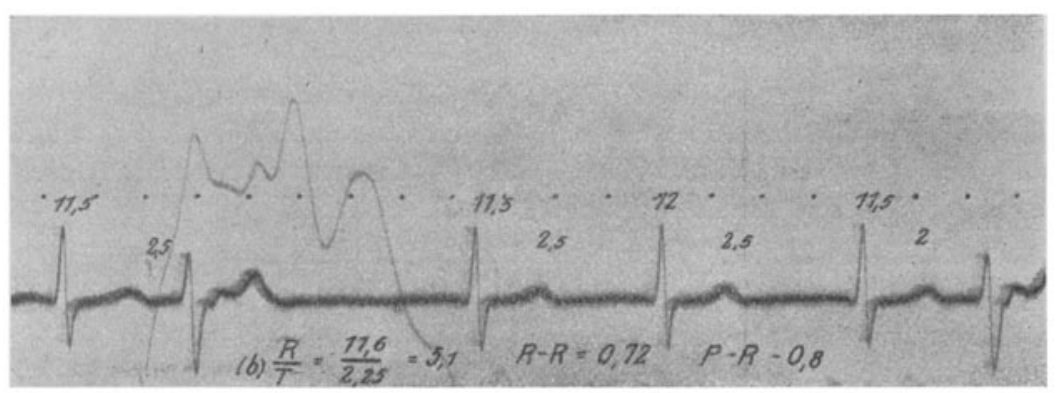

6 Minuten nach 1 dem ersten Koblensäurebade von $32^{\circ} ; 15$ Minuten Dauer.

In beiden Curven: Geschwindigkeit $\mathbf{4 0} \mathrm{mm}$ in der Secunde; Zeitschreibung 0,2 Secunden. Empfindlichkeit des Systems: $1 \mathrm{~mm}$ Ausschlag $=0,7 \cdot 10^{-8} \mathrm{~A}$.

15. 9. 1913. 1. Bad. Temperatur $32,5^{\circ}$. Dauer 15 Minuten.

Puls Blutdruck $I(\mathrm{~mm}) \quad \mathrm{F}(\mathrm{mm}) \quad \mathrm{I} / \mathrm{F}$ Abl.-Elektr. vor dem Bade . . . 96 regelm. $\quad 135-175 \quad 11,4 \quad 3,3 \quad 3,4$ $" \quad$ nach $" \quad$ ". . . stark unregelm. $140-175 \quad 11,6 \quad 2,25 \quad 5,1$ Siehe Gurve, Abb. 1.

Ergebnis. 10 Min. nach dem Bade: Bad schlecht vertragen, Herzklopfen, unregelmässiger Puls infolge gehäufter Bigeminie (vgl. Curve), der vorher rhythmische Puls ist irregulär geworden infolge zeitweise zwischen die normalen Perioden eingestreuter vorzeitiger Schläge vom Typus der Extrasystolen A. Keine wesentliche Aenderung des Blutdrucks. Elektro- 
cardiogramm: 10 Minuten nach dem Bade ist in den vergleichbar gebliebenen Normalperioden die Höhe der Initialzacke nicht verändert, aber die Finalschwankung ist stark erniedrigt, also der Quotient I/F grösser als vorher.

6. Po., Kaufmann, 24 Jabre. Klagen über Erregtheit, Müdigkeit, zeitweises Herzklopfen. Herz ohne krankbaften Befund. Lidflattern, Fingerzittern, gesteigerte Sehnenreflexe. Wassermannsche Reaction 0.

10. 10. 1913. 1. Bad. Temperatur 32,5 0. Dauer 15 Minuten.

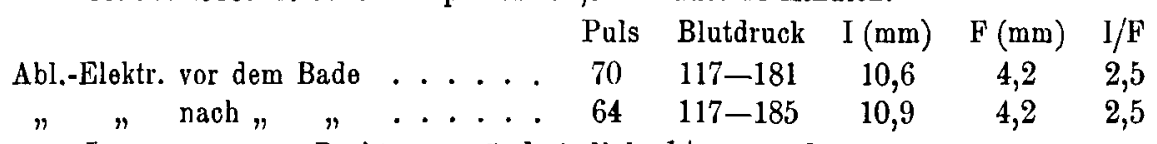

Lungengrenzen: Rechts unverändert, links $1 / 2 \mathrm{~cm}$ nach unten.

Ergebnis: Bad ohne jede Beschwerde vertragen. 10 Minuten nachher: Lungengrenzen kaum verändert, Puls kaum verlangsamt, Blutdruck unverändert. Elektrocardiogramm ist durch das Bad 10 Minuten später nicht beeinflusst.

Die drei letzten Beispiele zeigen in ihrem Verhalten gegenüber den Kohlensäurebädern eine andere Reaction als die ersten. Klinisch bilden sie cine gesonderte Gruppe, dortstanden im Vordergrunde dieZustände von Her z m us k elschwäche, hier wird das klinische Bild bestimmt von Beschwerden, die mit Störungen der Innervation der Kreislaufsorgane zusammenhängen.

Die Kranken mit Herz- und Gefässneurosen antworten anders auf den Keiz des Kohlensäurebades, als die mit einer Herzmuskelschwäche und intacter Innervation.

Der Fall von chronischem Alkoholismus (5), der mit starker Bigeminie und Herzbeschwerden noch 10 Minuten nach dem Bade reagierte, hatte keine Anzeichen einer besonders hoch einzuschätzenden Insufficienz des Herzmuskels, und sicher hatten einzelne der früher mitgeteilten Fälle erheblichere Kreislaufschwäche als er. Er bot keinen Herzbefund, der ihn an sich ungecignet für die Behandlung von vornherein erscheinen liess. Das Bad löste bei ihm einen offenbar rein nervös vermittelten Anfall von gehäufter Bigeminie aus. Dabei ist der Blutdruck kaum verändert, aber das Elektrocardiogramm zejgt neben der erwähnten Extrasystolie bei unveränderter lnitialzacke eine Verkleinerung der Finalschwankung, also ein anderes Verhalten als die Fälle von Herzmuskelschwäche und intacter Innervation.

Auch die übrigen Fälle dieser Gruppe von Herzneurosen zeigen kein einheitliches reactives Verhalten nach dem Bade. Die Bäder werden gut vertragen, aber ihr Einfluss ist unvollständig und wenig ausgiebig: so wird 10 Minuten nach dem Bade der Blutdruck nicht gesteigert gefunden, er ist, wenn überhaupt, nur vorübergehend beeinflusst worden. Regelmässiger ist anzutreffen die Verlangsamung des Herzschlages und die inspiratorische Einstellung der Lungengrenzen. Das Elektrocardiogramm bleibt in einem Falle unbeeinflusst, bei einem anderen Kranken sind die Erhebungen nach dem Bade niedriger gezeichnet, aber hier fällt auf, dass die Finalschwankung im Verhältnis mehr verkleinert ist als die erste Kammerzacke, und dass dadurch der Quotient I/F grösser geworden ist. 


\section{Die Aenderungen des Elektrocardiogramms durch das Bad in ihrer Beziehung zur Aenderung des Leitungswiderstandes der Haut.}

Der Analyse der Elektrocardiogramme ist bisher zu Grunde gelegt worden die Ausmessung der Ausschläge nach Millimetern Höhe. Es genügt aber nicht, auf diese Feststellung sich zu beschränken. Um die gewonnenen Werte einer Deutung zuzuführen, ist es geboten, eine weitere physikalische Controlle des Elektrocardiogramms einzuführen: die Bestimmung der Widerstände im Ableitungskreise vor und nach dem Bade. Das Niveau der Curven wird durch die Widerstände im Stromkreise bestimmt, und es frägt sich nun, wie weit die kühlen Kohlensüurebäder imstande sind, die elektrische Leitfähigkeit der Haut zu ändern. Die Leitfähigkeit hängt von der Durehfeuchtung der Epidermis $a b$, und diese wird im wesentlichen beherrseht von der Tätigkeit der Schweissdrüsen. Hier müsste wahrscheinlich der Einfluss der Kohlensäurebäder angreifen.

Diese Function und ihre Aenderung durch die Badeprocedur bei Herzschwächezuständen und bei Herzneurosen zubestimmen, ist an und für sich von klinischem Interesse, und ist ein nutzbarer Beitrag zur Analyse der Mechanik des Bades.

Kellermann (9) hat gefunden, dass Wärmeproceduren den Leitungswiderstand des menschlichen Körpers gegen den galvanischen Strom herabsetzen, während Kaltproceduren eher geeignet sind die Widerstände der Epidermis zu erhöhen. Die Methodik der Messung (20 Volt und polarisierbare Elektroden) bei der Prüfung sind ebenso, wie bei den früheren Untersuchungen übor den Leitungswiderstand der Haut (Stinzing, Jolly, Martius) zu sehr verschieden von der hier verwendeten, um eine Vergleichung der Ergebnisse ohne weiteres zu gestatten.

Darüber hinaus war es für die Deutung der Herzcurven geboten, diesen Weg zu gehen. Denn im Fortgang der Untersuchungen mehrten sich die Schwierigkeiten, aus den blossen Messungswerten gesetzmässige Beziehungen zu gewinnen. Das zeigte sich weiterhin bei der zweifachen Ableitung von Arm und Brust und der Vergleichung ihrer Werte und stellte sich als notwendig heraus bei der Prüfung von Kranken, deren Krankheitsbild und Reactionsweise durch ein nervöses Moment bestimmt wurde.

Der Elektrocardiograph, die bei diesen Untersuchungen verwendete Schleife des Apparates von Siemens\&Halske, ist ein Strom-Messinstrument. Die Schleife wird in der Technik als rasch und zuverlässig arbeitendes Ampèremeter bei Wechselstrommessung verwendet. Die Millimeter der Ausschlïge auf der Curve sind in Ampère ohne weiteres zu lesen, wenn der Ampìrewert eines Millimeterausschlages der Schleife bei der für den Versuch eingestellten Empfindlichkeit des Systems durch Eichung bestimmt ist.

Die Stromstärke und ihr graphischer Ausdruck, das Elektrocardiogramm, ist nach dem $0 \mathrm{hmschen}$ Gesetz $(\mathrm{e}=\mathrm{i} \cdot \mathrm{r})$ umgekehrt proportional dem Widerstande im Ableitungskreise. Die jedesmalige Bestimmung der Widerstände gibt also einen Anhalt dafür, in welchem Ausmasse Vergrösserungen oder Verkleinerungen nach dem Bade zu erwarten sind, und weiter ein 
Urteil, ob darüber hinaus und davon unabhängig, Beeinflussungen stattgefunden haben.

Das Siemens \& Halskesche Instrument enthält eine Messeinrichtung: an den Ableitungskreis wird eine Spannung von 1,5 Volt angelegt und die Stromstärke abgelesen, dann ist $\mathrm{r}=\mathrm{e} / \mathrm{i}$.

Wesentliche Störungen durch Polarisation kamen bei den benutzten Elektroden und der Controlle durch Stromwendung im besonderen Ausmasse nicht vor. In Fällen, wo der Zeiger des Ampèremeter bei der Einschaltung und Durchströmung nicht sofort ruhig stand, sondern je nach der Stromrichtung zunächst langsam stieg oder fiel, wurde nach dem Stillstand, zuweilen erst nach 2-3 Minuten, abgelesen, und bei verschiedenen Zahlen in verschiedener Stromrichtung der Mittelwert genommen. Grössere Schwierigkeiten entstehen zuweilen bei Basedow, aber diese Fälle werden in der Zusammenstellung hier nicht berücksichtigt.

Es müssen zu der Frage der Widerstandsbestimmung zunächst einige Bemerkungen gemacht werden. Es ist klar, dass der Wert, der als Widerstand der Haut bestimmt wird, zunächst unmittelbare Geltung nur hat für die Stromart und Stromstärke, mit der gearbeitet wird.

Die Grösse, die in Ohm berechnet und als Widerstand des im Kreise liegenden Menschen bezeichnet wird, wechselt je nach der angewendeten Methodik.

Für die Analyse des Elektrocardiogramms wäre der theoretisch richtige Wert derjenige, der mit Strömen gewonnen wird von ähnlicher Stärke, Spannung und Richtung, wie sie dem Elektrocardiogramm eignen und die sich auf den gleichen Bahnen bewegen. Ein solcher Wert ist zunächst ein Idealwert. Die Widerstandswerte, deren Ermittlung leicht und genau gelingt, werden mit Strömen gewonnen, die sich wesentlich von denen des Elektrocardiogramms unterscheiden. So auch die hier angewendete Messmethode. Es wurde ein constanter Strom von 1,5 Volt Spannung mit unpolarisierbaren Elektroden an den Ableitungskreis vom Menschen gelegt. Es ist dabei wohl zu bedenken, dass in dem complicierten Elektrolyten, dem menschlichen Körper und seinen Hautdecken mit ihren Eigenströmen, Reactionserscheinungen und elektrische Spannungen ausgelöst werden.

Aber diese Werte, die mit dem constanten Strom von 1,5 Volt Spannung erhalten wurden, haben dennoch einen relativen Wert. Den Beweis gibt ein Vergleich mit verschiedenen Messmethoden an demselben Menschen und unter gleichen Bedingungen. Verglichen wurde mit der Kohlrauschschen Telephonmethode mit schwachem Wechselstrom. Dabei ergab sich, dass die Werte ziemlich weit von einander entfernt lagen. Die Unterschiede sind wesentlich, Näheres bleibt einer weiteren Mitteilung vorbehalten.

Zweitens aber ergab sich, dass die bei verschiedenen Kranken gewonnenen Zahlen in einer recht genauen Proportion zu einander stehen, so dass man sagen kann, die Nulllinie ist bei beiden Messmethoden verschieden, aber die über derselben aufgetragenen Grössenwerte sind unter einander vergleichbar, denn sie verschieben sich in dem gleichen 
Verhältnis. Ein Vergleich der Ohmzahlen bei der einen und bei der anderen Messung bei verschiedenen Kranken gibt die gleichen Verschiebungen und zwar gleich nicht nur in der Richtung, sondern auch annähernd gleich in der Stärke wenigstens innerhalb der hier in Frage kommenden Ohmgrössen.

Für die in dieser Arbeit verwendeten Zahlen gilt also Folgendes:

Die Zahlen entsprechen nicht den Grössenwerten, die bei der Schreibung des Elektrocardiogramms als Widerstand anzunehmen sind, in absolutem Masse. Dagegen sind sie brauchbar im relativen Sinne zur Beurteilung der Richtung und des Umfanges, in der sich die Widerstände im Ableitungskreise durch das Bad verändert haben. Mehr wurde von diesen Werten bei diesen Untersuchungen nicht verlangt. Weitere Prüfungen werden darüber aufzuklären haben, welche der verschiedenen Messmethoden des Körperwiderstandes diejenige ist, die den wirklichen Verhältnissen bei der Schreibung des Elektrocardiogramms am nächsten kommt.

Die erhaltenen Werte für die Widerstände vor und nach dem Bade können einen gewisseu Anspruch auf Zuverlässigkeit machen. Für die vorliegende Frage sind sie geeignet, scheinbar unvereinbare Befunde zu klären und Beiträge zu liefern für die Hautreaction von Herzkranken.

Es seien einige in dieser Art untersuchte Fälle mitgeteilt.

7. Mü., Steinträger, 42 Jahre. Myodegeneratio cordis, Potatorium und schwere Körperarbeit. Seit 3 Wochen leichte Oedeme und Kurzatmigkeit bei der Arbeit. Herz nach beiden Seiten mässig verbreitert, systolisches Mitralgeräusch, Lebercirrhose, Pulsus irregularis perpetuus bei normalem Kammerelektrocardiogramm. Bei ruhigem Leben im Krankenbause keine Beschwerden.

17. 12. 1913. 1. Bad. Temperatur $32^{\circ}$. Dauer 15 Minuten.

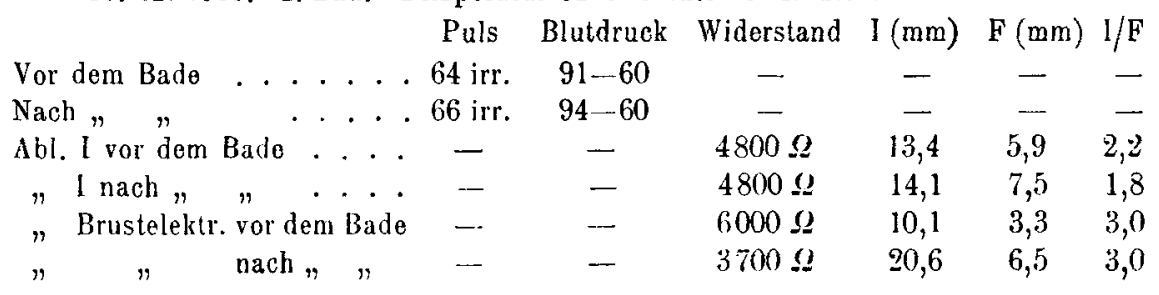

Ergebnis: Bad ohne Beschwerden vertragen. 10 Minuten nach dem Bade sind Puls und Blutdruck kaum beeinflusst. Beachtungswert ist die Verschiedenheit der Hautreaction an Arm und Brust, die Brusthaut reagiert stark durch Widerstandsverminderung, die Armhaut überhaupt nicht. Die Ursache ist vielleicht die jahrelang geübte Gewohnheit, mit entblössten Armen im Freien zu arbeiten.

Hieraus ergibt sich der Wert der Hautwiderstandsmessung für das Lesen des Elektrocardiogramms: bei Armableitung Initialzacken kaum verändert, bei Brustableitung verdoppelt im Sinne der Herabsetzung der Widerstände durch das Bad.

Als gute und zweckentsprechende Reaction der Haut muss nach weiter unten mitzuteilenden Befunden eine Erhöhung der Widerstände bezeichnet werden. In diesem Falle ist die Hautreaction eine nicht erwünschte. Die Fehlreaction dieses Bades zeigt sich ferner an in dem Elektrocardiogramm: die Finalschwankung ist in Ableitung I wenig höher 
geworden, in Abl.-Elektr. in dem gleichen Verhältnis erhöht wie die Initialzacke, so dass der Ventrikelquotient einmal nur einen wenig kleineren Wert zeigt als vor dem Bade, das andere Mal aber gleich geblieben ist.

Es lässt sich also sagen, dass das Kohlensäurebad, wie übrigens nicht selten gerade bei Lebercirrhose, eine unzweckmässige Reaction auslöst, und bei diesem Kranken eine nicht angezeigte Bäderprocedur ist, die keinen Erfolg verspricht. Der weitere klinische Verlauf bestätigte diese Ueberlegung.

Der nächste Fall zeigt die starke und nachhaltige Badewirkung am reactionsfähigen Kranken.

8. Kr., kräftiger Schankkellner, 23 Jahre. Starkes Potatorium, Myoinsufficienz, grosse Oedeme, schwere Cyanose bei der Aufnahme; durch Digitalisierung schnell gebessert. Seit 14 Tagen ohne Digitalis, bei rubiger Lebensweise keine Beschwerden (Curve $3 \mathrm{a}$ und $3 \mathrm{~b}$ ).

Curven $3 \mathrm{a}$ und $3 \mathrm{~b}$. Pat. Kr.

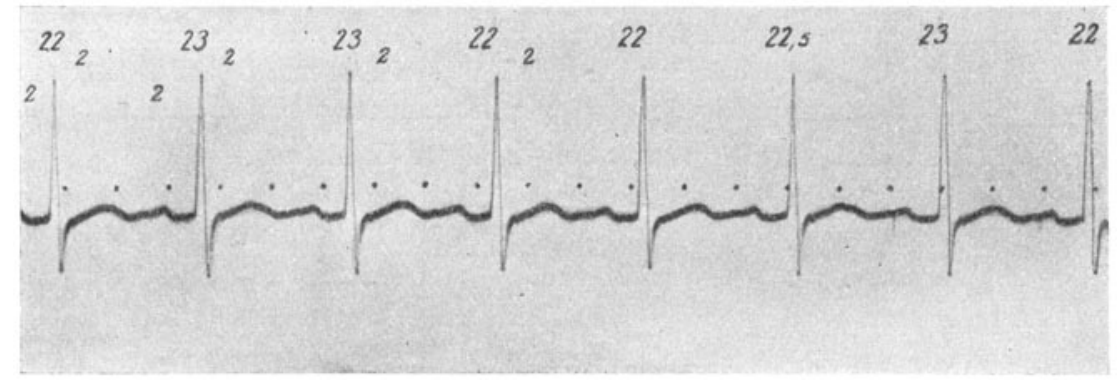

Vor dem Bade.

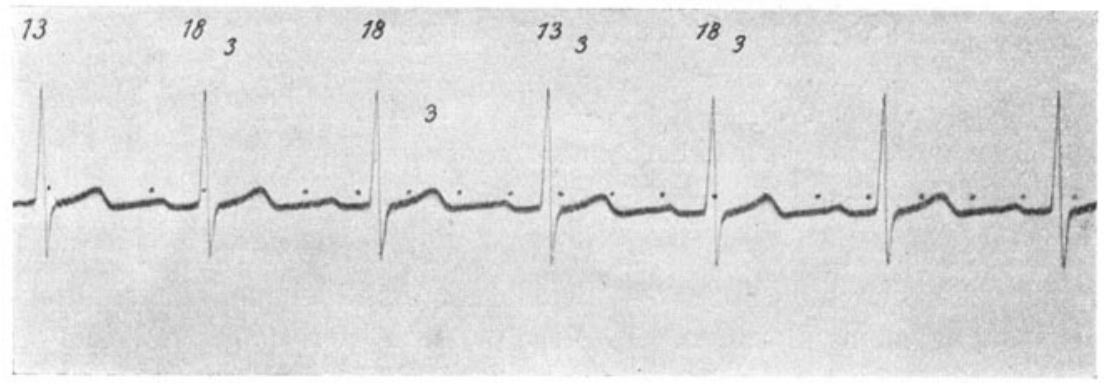

Nach dem zweiten Kohlensäurebade von $32^{\circ} ; 12$ Minuten Dauer.

In beiden Curven: Geschwindigkeit $40 \mathrm{~mm}$ in der Secunde; Zeitschreibung 0,2 Secunden. Empfindlichkeit des Systems: $1 \mathrm{~mm}$ Ausschlag $=0,0 \cdot 10^{-8} \mathrm{~A}$.

18. 12. 1913. 2. Bad. Temperatur $32^{\circ}$. Dauer $12 \mathrm{Min}$.

Puls Blutdruck Widerstand A (mm) I (mm) F(mm) I/F A-I $\begin{array}{lllllllll}\text { Vorher } & 96 & 150-160 & 6000 \Omega & 2 & 23 & 2 & 11,5 & 0,17 \text { Sec. } \\ \text { Nachher } & 80 & 164-180 & 8400 \Omega & 1,5 & 18,5 & 3 & 0 & 0,20\end{array}$ Nachher $80 \quad 164-180 \quad 8400 \Omega \quad 1,5 \quad 18,5 \quad 3 \quad 6 \quad 0,20$ "

Lungengrenzen rechts unverändert, links $1 \mathrm{~cm}$ nach unten verschoben.

Ergebnis: Bad gut vertragen. 10 Minuten nachher: Puls verlangsamt, Blutdruck gesteigert, Lungengrenzen nicht verschoben, Haut- 
widerstand erhöht. Elektrocardiogramm: Vorkammerschwankung und Initialzacke niedriger, Finalschwankung höher als vor dem Bade, also Ventrikelquotient kleiner geworden. Dabei ist zu beachten die Verlängerung der Ueberleitung von Vorkammer zu Kammer: A-I von 0,17 Sec. auf 0,20 Sec., also negative dromotrope Vaguswirkung.

Reactionen in dieser Stärke werden in der Regel nur von den ersten Bädern ausgelöst, im weiteren Verlauf schwächt die Wirkung sich ab, wofür auch dieser Fall einen Beleg gibt:

29. 12. 1913. 6. Bad. Temperatur $32^{\circ}$. Dauer 15 Min.

\begin{tabular}{|c|c|c|c|c|c|c|c|c|}
\hline & & Puls & Blutdruck & Widerstand & $\Lambda(\mathrm{mm})$ & $\mathrm{I}(\mathrm{mm}$ & $\mathrm{F}(\mathrm{mm})$ & $1 / F$ \\
\hline Abl.Elektr & vorher & 86 & $128-163$ & $6000 \Omega$ & 3 & 37 & 5,5 & 6,7 \\
\hline$n$ & nachher & 80 & $132-174$ & $7500 \Omega$ & 2 & 37 & 7,5 & 5 \\
\hline
\end{tabular}

Die Beeinflussung ist nicht so stark, wie oben beschrieben, aber im gleichen Sinne ausgefallen.

9. B., Reisender, 44 Jahr. Lebercirrhose, Alkobolismus chronicus. Seit 5 Jahren öfter Anfälle von Gelbsucht mit Schwellungen an den Füssen, im Leib. Leber und Milz vergrössert und bart fühlbar. Bei ruhiger Lebensweise beschwerdefrei.

9. 12. 1913. 1. Bad. Temperatur $33,5^{0}$. Dauer 12 Minuten.

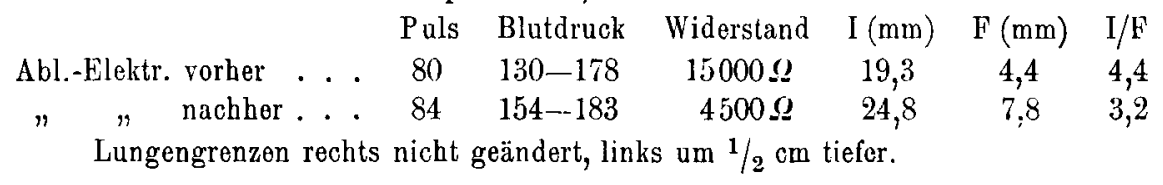

24. 1. 1914. 17. Bad. T'emperatur 32 \%. Dauer 15 Minuten.

$\begin{array}{cccccccc} & & \text { Puls } & \text { Blutdruck } & \text { Widerstand } & \mathrm{I}(\mathrm{mm}) & \mathrm{F}(\mathrm{mm}) & \mathrm{I} / \mathrm{F} \\ \text { Abl.-Elektr. vorher . . . } & 72 & 144-170 & 4700 \Omega & 18,8 & 5,0 & 3,7 \\ \text { " } & \text { " nachher . . . } & 72 & 140-177 & 8200 \Omega & 15,3 & 4,1 & 4,0 \\ \text { " I vorher . . . . . . } & - & - & 3700 \Omega & 25 & 10 & 2,5 \\ \text { "I nachber . . . . . } & - & - & 5300 \Omega & 21 & 8 & 2,6\end{array}$

Lungengrenzen beiderseits um $2 \mathrm{~cm}$ tiefer getreten.

Ergebnis: Die ersten Bäder werden schlecht vertragen, bei den späteren Gewöhnung an den Reiz. Während der fortgesetzten Bäderbehandlung bessern sich die Klagen - Beklemmungsgefühle und Herzangst und das allgemeine Befinden. 10 Minuten nach dem ersten schlecht vertragenen Bade eine anscheinend unzweckmässige Reaction: Puls rascher, aber Blutdruck gesteigert, Lungengrenzen nicht verschoben, der vorher sehr hohe Hautwiderstand nach dem Bade viel niedriger. Elektrocardiogramm: Dadurch beeinflusst sind beide Kammererhebungen nachher grösser als vorher, aber die Finalschwankung mehr, so dass I/F verkleinert.

10 Minuten nach dem 17. beschwerdelos vertragenen Bade: Puls und Blutdruck unbeeinflusst, dagegen der Hautreflex normal, d. h. die Widerstände erhöht. Elektrocardiogramm: dementsprechend erniedrigt und zwar in beiden Ableitungen. Diesmal hat sich aber die Finalschwankung noch mehr verkleinert und dadurch ist der Quotient $\mathrm{I} / \mathrm{F}$ grösser als vor dem Bade. 
Auch in diesem Falle von Lebercirrhose mit Herzschwäche erscheint angesichts des mangelhaften Herzreflexes der Nutzen der Badekur fragwürdig.

10. M., Locomotivführer, 37 Jahre. Mitral- und Aorteninsufficienz nach Gelenkrheumatismus vor 3 Jahren, seit 3 Monaten Herzklopfen und Kurzatmigkeit, keine Schwellungen. Wassermannsche Reaction 0.

24. 1. 1914. 3. Bad. Temperatur 33,50. Dauer 15 Minuten.

\begin{tabular}{|c|c|c|c|c|c|c|c|}
\hline & Puls & Blutdruck & Widerstand & $\mathrm{A}(\mathrm{mm})$ & $\mathrm{I}(\mathrm{mm})$ & $F(\mathrm{~mm})$ & $\mathrm{l} / \mathrm{F}$ \\
\hline Vorher & 86 & $136-140$ & $5300 \Omega$ & 2,6 & 28,8 & 4,8 & 6 \\
\hline Nachher. & 86 & $135-140$ & $6300 \Omega$ & 2,1 & 20,2 & 3,9 & 5 \\
\hline
\end{tabular}

Lungengrenzen beiderseits um $2 \mathrm{~cm}$ nach unten verschoben.

Ergebnis: Bäder gut vertragen. 10 Minuten nachher: Puls und Blatdruck gut vertragen, Lungengrenzen nicht verschoben. Widerstand erhöht. Elektrocardiogramm: daher in sämtlichen Erhebungen niedriger, da $\mathrm{F}$ weniger erniedrigt ist als $\mathrm{I}$, ist der Quotient $\mathrm{I} / \mathrm{F}$ kleiner als vorher.

In diesem Falle ist 10 Minuten nach dem Bade die Reaction der Haut und des Elektrocardiogramms nachweisbar, dagegen nicht die des Pulses und der Atmung, die also hier, wenn überhaupt beeinflusst, labiler ist.

11. Th., Lehrling, 16 Jahre. Neuropath, dauernd boschleunigter Puls, zeitweises Herzjagen. Kleines Herz im Röntgenbilde.

10. 1. 1914. 4. Bad. Temperatur $31^{\circ}$. Dauer 15 Minuten.

$\begin{array}{cccccccc} & & \text { Puls } & \text { Blutdruck } & \text { Widerstand } & \mathrm{I}(\mathrm{mm}) & \mathrm{F}(\mathrm{mm}) & \mathrm{I} / \mathrm{F} \\ \text { Abl.-Elektr. vorher. . . . } & 102 & 125-162 & 6600 \Omega & 18,1 & 4,6 & 4 \\ \text { " } \quad \text { " nachher . . . } & 104 & 125-165 & 7500 \Omega & 15,1 & 3,1 & 5 \\ \text { " I vorher. . . . . . . } & - & - & 5700 \Omega & 20,5 & 6,8 & 3 \\ \text { " I nachher . . . . . . } & - & - & 5900 \Omega & 18,5 & 6,0 & 3\end{array}$

Lungengrenzen links unverändert, rechts um $1 / 2 \mathrm{~cm}$ nach unten verschoben.

Ergebnis: Bad ohne Beschwerden vertragen. 10 Minuten nach dem Bade Puls und Blutdruck nicht beeinflusst. Elektrocardiogramm: entsprechend der Zunahme des Hautwiderstandes niedriger und zwar in Abl. I in beiden Kammererhebungen gleichsinnig, also I/F unverän dert geblieben, in Abl.-Elektr. nach dem Bade F kleiner als I, daher $\mathrm{I} / \mathrm{F}$ g rösser geworden.

In diesem Falle von Hermeurose ist 10 Minuten nach dem Bade abgesehen von der Hautreaction eine Beeinflussung nicht nachweisbar.

Alle die von der Reaction des Herznervensystems abhängigen Aenderungen werden vermisst; aus diesem Fehlen einer Beeinflussung ergibt sich der Schluss, dass der Kranke für eine Behandlung mit Kohlensäurebäder ungecignet ist, was auch die klinische Beobachtung zeigte.

Bei der letzten Reihe von Beispielen waren bei der Aufnahme des Elektrocardiogramms die Widerstände im Ableitungskreise bestimmt worden.

Mit der Messmethode wurden bei den verschiedenen Kranken für den Leitungswiderstand Werte von recht verschiedener Grössenordnung ermittelt. Jeder Kranke hat seine individuellen Werte und seine individuellen Schwankungen.

Die Bestimmung der Widerstände ist wichtig, denn es zeigt sich durchgehends, dass sie die Höhe der Ausschläge des Elektrocardio- 
gramms ganz wesentlich bestimmen. Es zeigt sich weiter, dass die Kohlensäurebäder eben diese Hautwiderstände fast regelmässig und in starker und nachhaltiger Weise beeinflussen.

Zumal bei Kranken mit Herz- und Gefässneurosen und bei solchen, die das Bad schlecht vertragen, werden ungewöhnliche Grössenänderungen des Elektrocardiogramms aufgezeichnet, die verständlich werden, sobald das ungewöhnliche Verhalten der Hautwiderstände dabei berücksichtigt wird. So war in einem Falle der Widerstand vor dem Bade abnorm gross und in anderen Fällen wurde er durch die Procedur im Sinne eines "Abreagierens" ${ }^{*}$ geändert und auffallend verkleinert. Unerwartete Aenderungen des Elektrocardiogramms bei manchen Kranken mit chronischem Alkoholismus und mit vasomotorischen Neurosen werden durch diese Hautreaktionen erklärt.

Wird mit verschiedenen $\Lambda$ bleitungen an demselben Kranken gearbeitet, so ist, wie die Erfahrung lehrt, mit der Möglichkeit zu rechnen, dass über der Brust und an den Armen der Hautwiderstand nicht im gleichen Sinne verändert wird, was dann ungleichsinnige Aenderungen der beiden Elektrocardiogramme zur Folge hat.

Jedenfalls zeigen die mitgeteilten Beispiele, dass das kühle Koblensäurebad die ausgesprochene Eigenschaft hat, bei vielen Kranken den Leitungswiderstand der Haut nachhaltig und noch 10 Minuten nachher zu beeinflussen. In dieser Beeinflussung spiegelt sich wieder die Aenderung der Function der Schweissdrüsen, von der die Durchfeuchtung der Epidermis wohl im wesentlichen abhängt. So reiht sich die Reaction auf die Leitfähigkeit der Haut als ein bedeutsames Glied in die Reihe der übrigen Reactionen des Bades ein.

Die Abhängigkeit von dieser Reaction zeigen sämtliche Erhebungen des Elektrocardiogramms, aber am besten das elektrische Aequivalent der Vorhofzuckung und die erste Kammerzacke, während bei der Finalschwankung andere ändernde Einflüsse vielfach überwiegen. Auf dieses Hervortreten besonderer ändernder Einflüsse auf die zweite Kammererhebung weist ja bereits die Veränderung hin, die der Quotient I/F nach dem Bade zeigte.

\section{Anfstellung verschiedener Reactionstypen und deren Bedingtheit durch die Art der nervősen reflectorischen Anspruchsfähigkeit des Herzens und der Gefüsse.}

Auf Grund der an Herzkranken gewonnenen Erfahrungen lassen sich aus den geprüften Functionen gewisse Reactionsformen aufstellen und zu Reactionstypen ordnen. Als solche lassen sich charakterisieren: Die Reactionsweise der für die Behandlung im klinischen Sinne gut geeigneten Fälle von Herzmuskelschwäche. Hier ist wieder zu unterscheiden die Beeinflussung darch die ersten Bäder und durch die späteren Bäder. Das Bad löst hier, wenn auch in wechselnder Stärke, so doch in bestimmter Richtung eine Reihe von Reflexen aus. Davon ist abzugrenzen diejenige Form der Beeinflussung, die sich bei Kranken findet, deren klinisches Bild von nervösen Störungen der Kreislaufsorgane bestimmt 
wird. Hier werden die nervösen Reflexe nicht nur unvollständig und wenig nachhaltig, sondern auch vielfach nach anderer Richtung ausgelöst. Es ist selbstverständlich, dass bei der häufigen Vereinigung organisch und nervös bedingter Herzbeschwerden die Typen sich vielfach verwischen. Man hat ferner zu berücksichtigen, dass ein ungewöhnlicher Reactionsverlauf und eine Umformung der Reflexe auch bei denjenigen Kranken mit schwachem Herzmuskel zu erwarten ist, bei denen die durch das Bad gesetzte Anstrengung die Schwelle der Leistungsfähigkeit des Gefässapparates überschreitet.

Einer im klinischen Sinne günstigen Beeinflussung des Kranken entspricht etwa der folgende Reactionstypus:

Innerhalb der Frist von etwa 10 Minuten nach einem wirksamen kühlen Kohlensäurebade ist der Puls um 10-20 Schläge in der Minute verlangsamt, der Blutdruck um 10-20 mm Quecksilber erhöht. Die unteren vorderen Lungengrenzen sind um etwa $2 \mathrm{~cm}$ nach unten verschoben. Die mittlere Atmungsstellung der Lungen ist eingestellt auf eine inspiratorische Dauerstellung; der durch den Reflex auf das $\Lambda$ temcentrum vermittelte Tiefstand des $Z$ werchfells hat eine Aenderung der Herzlage zur Folge, die Herzachse wird steiler gestellt und mehr nach vorn gerichtet.

Der galvanische Leitungswiderstand der Haut ist grösser geworden, bis zu 50 pCt. des ursprünglichen Wertes.

Das Elektrocardiogramm spiegelt diese Widerstandserhöhung im Ableitungskreise wider und zeigt eine kleinereVorkammerzacke und eine kleinere erste Kammerzacke. Dieser im Sinne einer Verkleinerung wirkende Einfluss des Bades wird aber bei der zweiten Kammererhebung, der Finalschwankung, überkompensiert. Denn die Finalschwankung ist entweder absolut grösser als vor dem Bade oder sie ist wenigstens im Verhältnis zur Aenderung der übrigen Erhebungen des Elektrocardiogramms vergrössert. - Die typische Wirkung des Kohlensäurebades bei einem klinisch günstig beeinflussten Herzkranken ist also die Vergrösserung der Finalschwankung am Elektrocardiogramm.

Die Erhöhung der Finalschwankung zeigen Herzkranke ebenso wie Herzgesunde nach einer zweckmässig dosierten körperlichen Arbeit, doch ist sie dabei meist verbunden mit einer Verminderung des Hautwiderstandes wohl infolge der Anregung der Schweissdrüsentätigkeit. Das Elektrocardiogramm verzeichnet dann eine Vergrösserung sämtlicher Erhebungen gleichzeitig mit der bedeutenderen Erhöhung von F. Weiterhin unterscheidet sich die spezifische Badewirkung von der gewöhnlichen Körperübung dadurch, dass sie einen starken Vagusreiz am Herzen auslöst. Diese nachhaltige reflectorische Vagusreizwirkung zeigt sich nicht nur in der negativ chronotropen Componente der Herzwirkung des Vagus an, sondern auch in einer negativ dromotropen auf die Ueberleitung von Vorhof zu Kammer, die verzögert wird.

Aus den Untersuchungen ergibt sich weiterhin, dass die geschilderten typischen Beeinflussungen nach den ersten Bädern am stärksten sind, was auch Waledinsky bereits in seinen Tierversuchen gefunden 
hatte. Im Laufe einer länger fortgesetzten Bäderreihe werden in der Regel die Unterschiede vor und nach dem Bade geringer, die gleiche Procedur löst dann weniger energische und weniger nachhaltige Reflexe aus. Die Abstumpfung und Gewöhnung 'zeigt sich ebenso am Elektrocardiogramm wie an den übrigen geprüften Funetionen, jedoch in ungleichem Masse. So reagiert der Hautwiderstand immer wieder in der früheren Stärke, ebenso das Atemcentrum mit seiner inspiratorischen Einstellung der unteren Lungenränder. Dagegen lassen die Her'zwirkungen an Stärke und Dauer nach: es vermindern sich die Vagusreizwirkungen, ferner auch die Blutdrucksteigerung und am Elcktrocardiogramm die Höhenzunahme der zweiten Kammerschwankung.

Abweichungen von dem geschilderten Verhalten treten, soweit die Beobachtungen reichen, häufig auf bei Herzkranken, die in ihrem nervösen Reflexmechanismus auch sonst Abweichungen vom normalen Verhalten boten. Durch nervöse Labilität fallen unter den hier untersuchten Herzkranken einzelne jugendliche Kranke mit Klappenfehlern auf und ältere Kranke mit Lebercirrhose.

So hatte ein Kranker, dessen geklagte Beschwerden und dessen Bigeminie nach dem Bade erheblich zugenommen hatten, keine Widerstandserhöhung und zugleich eine erniedrigte Finalschwankang. $\Lambda$ ber es gehen in diesen Fällen subjective Empfindungen und objectiver Befund nicht immer Hand in Hand. Zuweilen war in diesen Fällen 10 Minuten nachber cine geringe Elektrocardiogrammreaction nachweisbar, aber es fehlten Blutdrucksteigerung und Pulsverlangsamung, so bei den Kranken mit Aorteninsufficienz. Gegensätzliche Ausschläge gaben dic reinen Fülle von Herzneurose. Man kann hier des öfteren von einem Abreagieren sprechen. Das äussert sich einmal in der subjectiven Reaction: das Bad ist unbekömmlich, als seine Folge werden Erregtheit und schlechter Schlaf angegeben. Objectiv werden 10 Minuten nach dem Bade deutliche und einheitliche Wirkungen an Puls, Blutdruck und Elektrocardiogramm nicht angetroffen. Nur der Hautwiderstand ist in der Regel, wic es der Norm entspricht, erhöht, bisweilen aber an den verschiedenen $\Lambda$ bleitungsstellen ungleichmässig beeinflusst; am Elektrocardiogramm findet man an der Finalschwankung keine oder keine nennenswerten Grössenverschiebungen gegenüber den übrigen Zacken.

Im allgemeinen lässt sich aus den Untersuchungen feststellen, dass zwischen dem klinisch zu beobachtenden günstigen Einfluss des Bades und der Stärke der geprüften Reactionen, im besonderen auch des Elektrocardiogramms, ein gewisser $\mathrm{Zu}$ sammenhang besteht. Bei organisch Herzkranken, die Nutzen von dem Bade haben, lassen sich typische Reactionserscheinungen feststellen, bei den anderen Kranken dagegen, und vornehmlich bei den Herzneurosen werden diese Reactionen nicht oder gering ausgeprägt oder gegensätzlich geändert gefunden. In diesem Zusammenhange erweist sich das Elektrocardiogramm mit seiner Aenderung der Finalschwankung als ein gewisser Massstab für das Eintreten der Herzwirkung des Koblensäurebades. 


\section{Das Verhältnis der Voltzahlen der Elektrocardiogramme vor und nach dem Bade.}

Es ist gezeigt worden, dass an den Aenderungen der Vorkammerzacken und an den Höhenunterschieden der Kammeranfangszacken eine äussere Bedingung der Schreibung, nämlich der jeweilige Ableitungswiderstand, mit besonderer Deutlichkeit zum Ausdruck kommt. Die Widerstandsünderung ist aber nicht der einzige mittelbar beeinflussende Umstand, mit dem bei der Ableitung zu rechnen ist.

Daneben ist noch für eine grössere Zahl der Fälle zu berücksichtigen, dass durch das Bad der Zwerchfellstand erniedrigt wird, wodurch die Lage des Herzens zu den Ableitungsstellen sich mehr oder weniger verschiebt.

Der Anteil der Widerstandsänderung kann nun für die vergleichende Betrachtung dadurch ausgesehaltet werden, dass aus dem Product Ampèrezahl und Ohmzahl die Voltzahlen des Elektrocardiogramms dargestellt und in die Rechnung gesetzt werden.

Der mit dem constanten Strom von 1,5 Volt Spannung ermittelte Widerstand der Hautdecken entspricht nicht der Olımzahl, bei der das Elektrocardiogramm des Kranken geschrieben worden ist. Auch das Product aus der so gewonnenen Ohmzahl mit der Ampèrezahl der Zacken ist nicht die wahre Voltzahl des Elektrocardiogramms, aber es gestattet für den Vergleich vor und nach dem Bade einen Schluss auf die Richtung und die Stärke, in der sich die Voltzahlen gegen einander verschoben haben.

Unter dieser Beschränkung schien es von Nutzen für die Analyse des Elektrocardiogramms, bei einigen der untersuchten Kranken diese (relativen) Voltwerte zu berechnen und nebeneinander zu stellen.

I. Starke Beeinflussung des Elektrocardiogramms bei klinisch ausgiebiger Badreaction.

Kr., 18. 12. 1913. Myoinsufficienz.

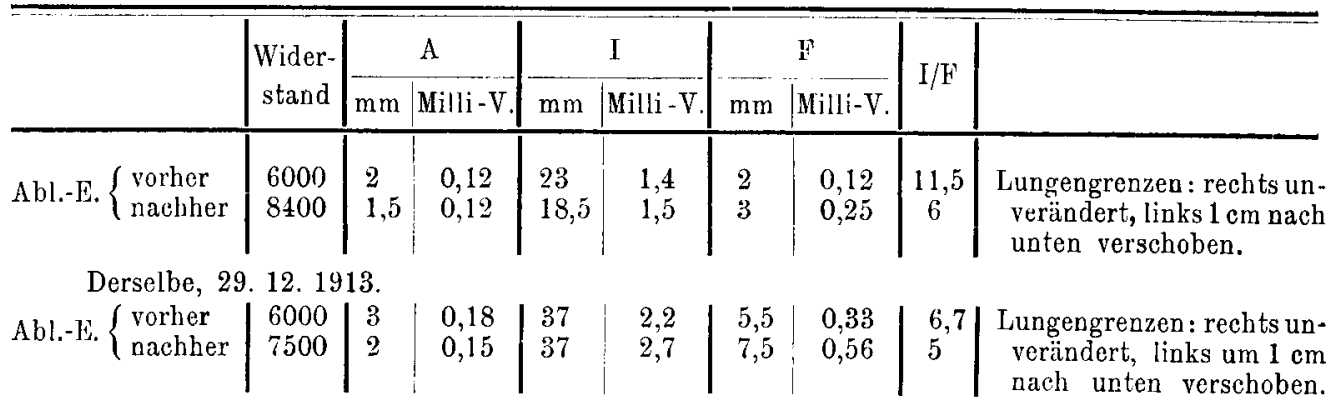

\section{Mässige und unvollständige Beeinflussung des Elektrocardiogramms.}

Be. Aeltere Lebercirrhose und Myoinsufficienz.

\begin{tabular}{|c|c|c|c|c|c|c|c|c|c|}
\hline Abl.-E. $\left\{\begin{array}{l}\text { vorher } \\
\text { nachber }\end{array}\right.$ & $\begin{array}{l}4700 \\
8200\end{array}$ & - & - & $\begin{array}{l}18,8 \\
16,3\end{array}$ & $\begin{array}{l}0,9 \\
1,3\end{array}$ & $\begin{array}{l}5,0 \\
4,0\end{array}$ & $\begin{array}{l}0,23 \\
0,32\end{array}$ & $\begin{array}{l}3,7 \\
4,0\end{array}$ & Lungengrenzen: beider- \\
\hline$\Lambda$ bl. I $\left\{\begin{array}{l}\text { vorher } \\
\text { nachher }\end{array}\right.$ & $\begin{array}{l}3700 \\
5300\end{array}$ & - & - & $\begin{array}{l}25,0 \\
21,0\end{array}$ & $\begin{array}{l}0,94 \\
\mathbf{1}, 1\end{array}$ & $\begin{array}{r}10,0 \\
8,0\end{array}$ & $\begin{array}{l}0,37 \\
0,42\end{array}$ & $\begin{array}{l}2,5 \\
2,6\end{array}$ & $\begin{array}{l}\text { seits um } 2 \mathrm{~cm} \text { nach } \\
\text { unten verschoben. }\end{array}$ \\
\hline
\end{tabular}

Me. Aorten- und Mitralinsufficienz. Beginnende Myoinsufficienz.

Abl. I \begin{tabular}{l|l|l|l|l|l|l|l|l|l} 
vorher & 5300 & 2,6 & 0,13 & 28,8 & 1,5 & 4,8 & 0,25 & 6 & Lungengrenzen: beider- \\
nachher & 6300 & 2,1 & 0,13 & 20,2 & 1,2 & 3,9 & 0,24 & 5 & seits um 2 cm nach unten \\
& & & & & & & & &
\end{tabular} 


\section{Geringe Beeinflussung des Elektrocardiogramms bei klinisch schlechter und unbekömmlicher Reaction.}

Th., Jugendliche Herzneurose.

\begin{tabular}{|c|c|c|c|c|c|c|c|c|c|}
\hline & \multirow{2}{*}{$\begin{array}{l}\text { Wider- } \\
\text { stand }\end{array}$} & \multicolumn{2}{|r|}{ A } & \multicolumn{2}{|r|}{ I } & \multicolumn{2}{|r|}{$\mathbf{F}$} & \multirow{2}{*}{$\mathrm{I} / \mathrm{F}$} & \\
\hline & & $\mathrm{mm}$ & Milli-V. & $\mathrm{mm}$ & Milli-V. & $\mathrm{mm}$ & Milli-V. & & \\
\hline Abl-E $\{$ vorher & 6600 & - & - & 18,1 & 1,0 & 4,6 & 0,27 & 4 & \\
\hline ADI.-E. nachber & 7500 & - & - & 15,1 & 1,0 & 3,1 & 0,21 & 5 & $\begin{array}{l}\text { Lungengrenzen : links un- } \\
\text { verändert, rechts } 1 / \mathrm{cm}\end{array}$ \\
\hline Abl. I $\left\{\begin{array}{l}\text { vorher } \\
\text { nachher }\end{array}\right.$ & $\begin{array}{l}5700 \\
5900\end{array}$ & - & - & $\begin{array}{l}20,5 \\
18,2\end{array}$ & $\begin{array}{l}1,0 \\
0,96\end{array}$ & $\begin{array}{l}6,8 \\
6,0\end{array}$ & $\begin{array}{l}0,3 \\
0,3\end{array}$ & $\begin{array}{l}3 \\
3\end{array}$ & nach unten verschoben. \\
\hline
\end{tabular}

Diejenigen Zacken des Elektrocardiogramms, die ihre versehieden grossen Ausschläge vor und nach dem Bade zu annähernd gleich grossen Voltwerten ergänzen, kennzeichnen sich dadurch als wesentlich abhängig von dem Wechsel der Leitungswiderstände. An den Stellen, wo verschiedene Ampirewerte bei der Berechnung auf ihre Voltzahl nahezu übereinstimmende Grössen ergeben, sind Zuwachs und Abnahme der Zackenhöhe als eine Function des Widerstandes erwiesen.

Bei den Fällen, die auf das Bad mit "Reaction" antworten, lassen sich die folgenden Beziehungen der entsprechenden Voltzahlen zu einander ableiten:

Die Vorkammern zeigen keine oder nur geringe $\Lambda$ b weichungen ihrer Voltzahlen vor und nach dem Bade. Die versehieden grossen Ausschläge auf dem Elektrocardiogramm sind also im wesentlichen verursacht durch den infolge des Bades veränderten und zwar in diesem Falle erhöhten Leitungswiderstand der Haut. Eine unmittelbare Beeinflussung der Vorkammerausschläge durch das Bad ist in diesen Fällen nur in geringem Masse eingetreten.

Ein ähnliches Verhalten zeigen die Anfangszacken der Kammern, doch ist bei ihnen häufiger eine Zunahme der Voltzahlen gegeben. Bei der Wertung dieses Zuwachses darf man nicht unberücksichtigt lassen, dass neben der Widerstandsänderung noch ein anderer, gleichfalls mittelbar wirkender Einfluss des; Bades hierin zum Ausdruck kommen kann, nämlich die durch das Bad veränderte Herzlage. Mit dieser Möglichkeit muss vor allem in denjenigen Fällen gerechnet werden, wo das Zwerchfell nach Ausweis der Percussion der unteren Iungengrenzen nach unten verschoben worden war.

Infolge der Einstellung des Zwerchfells auf eine tiefere respiratorische Mittellage wird die Herzlage verändert: die Längsachse wird steiler gestellt, und mehr nach vorn gedreht. Die Herzmasse steht vorher und nachher in einem anderen Verhältnis zu den Ableitungsstellen, und die von der Herzoberfläche an die Körperoberfläche abgeleiteten Potentialdifferenzen, die das Elektrocardiogramm zusammensetzen, werden abgeändert. Dadurch können die Ampère- und die Voltzahlen in Richtung und Stärke verändert werden. Die Beurteilung ist natürlich besonders schwierig beim pathologischen Herzen. So hatte ein Kranker mit Aorteninsufficien\% nach dem Bade eine Verkleinerung der Voltwerte beider Kammerzacken, zumal der Initialzacke. Aber dieser Fall zeichnet sich durch 
eine unzureichende Reaction aus, denn es fehlten Blutdrucksteigerung und Pulsverlangsamung, und nachweisbar waren nur Widerstandserhöhung der Haut und tieferer Stand des Zwerchfells, so dass der Fall vielmehr als ein Beispiel für den Einfluss der tieferen Einstellung des Zwerchfells auf die Voltzahlen des Elektrocardiogramms gelten kann. Unter diesen Umständen erscheint es angezeigt, diejenigen Fälle in erster Linie zu beachten, bei denen die Lungengrenzen sich nicht wesentlich verschoben haben.

In einem Falle mit kräftiger Badereaction und fast unverändertem Zwerchfellstand war die Voltzahl der Initialzacke etwas grösser geworden. Ueber das Verhalten dieser Zacke wäre etwa Folgendes zu sagen: Dieser Teil der Curve des Kammer-Elektrocardiogramms bringt in seinen Höhenunterschieden vorwiegend den Wechsel der Leitungswiderstände zum Ausdruck. Daneben aber zeigt der Vergleich der Voltzahlen und die Feststellung eines geringen Voltzuwachses, dass auch eine gewisse unmittelbare Beeinflussung durch das Bad vorhanden ist, die zwar nicht bedeutend ist, aber doch eine am Herzen selbst ausgelöste Badewirkung anzeigen dürfte.

Dieses gilt nun ganz besonders für die Endschwankung der Kammer. Bei der Schreibung des Elektrocardiogramms wirkt die Widerstandszunahme auf die Höhe sämtlicher Zacken erniedrigend ein, dagegen scheint die unmittelbare und eigentliche Herzwirkung des Bades, zumal für die líndschwankung der Kammer, nach der entgegengesetzten Richtung zu gehen, nämlich im Sinne einer Zunahme der Stromstärke. Bei der Endschwankung ist der Zuwachs so bedeutend, dass er den Einfluss der Widerstandszunahme übercompensiert und schon bei der Schreibung des Elektrocardiogramms auf der Curve in die Augen fällt. Sehr deutlich zeigt sich dieser Zuwachs bei der Betrachtung der Voltwerte, die ja unabhängig sind vom Wechsel des Widerstandes. Die Voltzahlen der Finalschwankung vor und nach dem Bade entfernen sich weit von einander. Bei kräftiger Badereaction beträgt die Erhöhung bis zu 100 pCt. des ursprünglichen Wertes. Diese stark erhöhten Voltzahlen werden u. a. auch bei solchen Herzkranken gefunden, bei denen die unteren Lungengrenzen nach dem Bade nicht verschoben sind; eine Aenderung der Herzlage darf also nicht verantwortlich gemacht werden für Erzeugung dieses Zuwachses.

Bei den Fällen von Herzneurose, bei denen die Reaction ausblieb, sind die Voltzahlen für sämtliche Teile des Elektrocardiogramms nicht erhöht und zuweilen etwas erniedrigt worden. Auf der Curve sind alle Zacken niedriger geschrieben. Aber darin kommt nichts weiter zum Ausdruck als die Zunahme der Widerstände im Ableitungskreise. Die Erhöhung der Voltzahl der Finalschwankung wird in diesen Fällen in der Regel vermisst.

In dem letzten Teil dieser Arbeit wurde in die Analyse des Elektrocardiogramms die Berechnung der relativen Voltzahlen eingeführt. Das hat dazu geführt, $z$ weierlei Arten von Beeinflussungen des Elektrocardiogramms durch das Bad zu unterscheiden und abzugrenzen. 
Einmal die Becinflussung durch äussere Bedingungen: die Widerstandszu - oder - abnahme im Ableitungskreis, dic die Zackenhöhe im umgekehrten Sinne ändert, und ferner die Verschiebung der Herzlage infolge der tieferen Einstellung des Zwerchfells nach dem Bade. Durch die Einführung der Voltzahlen an die Stelle der mm-Zackenhöhe konnten unter Berücksichtigung des Einflusses einer Lageveränderung des Herzens diejenigen Aenderungen des Elektrocardiogramms näher umgrenzt werden, in denen die unmittelbare und eigentliche Badewirkung zum Ausdruck kommt.

In den zur Untersuchung gelangten Fällen von Erkrankungen des Herzens wurden als unmittelbare Beeinflussungen bestimmt ein geringer Zuwachs der Voltzahl der Initialzacke und ein starker Zuwachs der Voltzahl der Finalschwankung. Diese Zunahme der Voltzahlen wurde in der Mehrzahl der auf Kohlensäurebäder kräftig ansprechenden Kranken gefunden. Die Herzcurve zeigte die Zeichen der Vagusreizung, die mehr oder weniger starke Verlangsamung der Schlagfolge und die Verzögerung der Reizleitung zwischen Vorkammern und Kammern.

Diese unmittelbaren Aenderungen des Elektrocardiogramms werden hervorgebracht durch die Erregung nervöser Herz- und Gefüssreflexe im Bade und überdauern die Badeprocedur um eine gewisse Zeit. Diese Art der Erregung setzt eine normale Anspruchsfähigkeit der Herz- und Gefässnerven voraus.

Sic kommt daher nicht oder nur unvollständig zustande bei Kranken mit abnormer nervös-reflektorischer Anspruchsfähigkcit.

\section{Zusammenfassung.}

Bei Kranken mit Herzmuskelschwäche verschiedener Ursache und bei Kranken mit vorwiegend nervösen Herzbeschwerden wurden unmittelbar vor und bis 10 Minuten nach einem kohlensauren Bade Elektrocardiogramm, Blutdruck, Leitungswiderstand der Haut und Zwerchfellstand bestimmt.

Für die klinische Betrachtung ergab sich dabei, dass die Reactionsweise der Kranken, genügende Kraft des Herzmuskels vorausgesetzt, wesentlich bestimmt wurde durch die Art der Anspruchsfähigkeit ihrer nervösen cardialen und vasomotorischen Reflexe.

Bei der Analyse des Elektrocardiogramms wurde der Versuch gemacht, zu unterscheiden zwischen den Veränderungen durch äussere Bedingungen: Widerstandszu- oder - Abnahme im Ableitungskreis und Verschicbung der Herzlage durch die tiefere Einstellung des Zwerchfolls, und zwisehen Aenderungen durch innere Bedingungen, den unmittelbaren und eigentlichen, durch nervöse Reflexmechanismen am Herzen ausgelösten Zustände.

Der Hautwiderstand wurde bestimmt gegen einen constanten Strom von 1,5 Volt Spannung. Die aus den Ampèrewerten der Zacken des Elektrocardiogramms and den Widerständen berechneten Voltzahlen geben nicht die absoluten Voltwerte des Elektrocardiogramms, aber sie gestatten 
einen Schluss auf dic Richtung und Stärke, in der sich dic Voltzahlen gegeneinander verschoben haben. Gelang es damit die äussere Bedingung: Widerstandswechsel aus der Vergleichung auszuschalten, so war es nicht möglich, den Einfluss der Lageveränderung bei den mannigfaltigen Bedingungen zu fassen, die bei der Drehung und Senkung pathologisch veränderter Herzen durch tiefere Einstellung des Zwerchfells möglich sind.

Bei der Aufstellung eines Reactionstypus wurden daher die Fälle mit unveränderten unteren Lungengrenzen besonders beachtet. Herzkranke ohne besondere nervöse Störungen und mit. zureichendem Herzmuskel zeigen etwa 10 Minuten nach dem Bade die folgenden Reactionen: Blutdrucksteigerung, häufig (bei fehlender Lungenstarre) Tiefertreten der unteren Lungengrenzen, erhebliche Zunahme des Leitungswiderstandes der Haut. In Abhängigkeit von der Widerstandszunahme auf der Curve des Elektrocardiogramms Erniedrigung der Vorkammerzacke und der ersten Kammerzacke, dagegen Erhöhung der Endschwankung der Kammer.

Die Voltzahlen sind für die Vorkammern nahezu unverändert, für die Initialzacke ein wenig erhöht, für die Finalschwankung bedeutend erhöht.

In diesem Verhalten der Voltzahlen kommt die unmittelbare und eigentliche Herzwirkung des Bades im Elektrocardiogramm zum Ausdruck. Hierher gehört auch die Verlangsamung der Schlagfolge und die Verzögerung der Reizleitung zwisehen Vorkammern und Kammern. Bei den untersuchten Herzkranken waren die bezeichneten Reactionen durchaus nicht immer vollständig ausgebildet. Da wegen zu grosser Herzschwäche ungeeignete Kranke nicht gebadet wurden, so schien die unvollkommene Ausprägung der Reaction weniger mit der organischen Erkrankung als mit einer abweichenden nervösen Reflexerregbarkeit der Kranken in Zusammenhang zu stehen.

Kranke mit rein nervösen Herzbeschwerden zeigen etwa 10 Minuten nach dem Bade: gewöhnlich Erhöhung der Hautwiderstände, aber manchmal in ungleicher Stärke an Brust und Armen, zuweilen tiefere Einstellung des Zwerchfells. In der Regel fehlen Blutdrucksteigerung und Pulsverlangsamung. Die Curve des Elektrocardiogramms zeigt bei Widerstandszunahme eine gleichmässige Erniedrigung sämtlicher Zacken und zumeist kaum veränderte Voltwerte; im wesentlichen also nur mittelbare Beeinflussungen des Elektrocardiogramms.

\section{Literatur.}

1. Nicolai, Ueber den Einfluss verschiedener Bäder auf das Herz. Med. Klinik. 1912. Nr. 21.

2. Rheinboldt und Goldbaum, Diese Zeitschr. 1911. Bd. 9.

3. A. Bickel, Physiologische Untersuchungen zur Bäderwirkung auf den Kreislauf. Med. Klinik. 1912. Nr, 24.

4. Rheinboldt, Ueber die Beeinflussung des Kaninchenelektrocardiogramms durch einfache Bäder und 0zetbäder von indifferenter Temperatur. Zeitschr.f: Balneologie. Bd. 4. Nr. 23. 
216 Brandenburg u. Laqueur, Aenderung. d. Elektrocardiogramms v. Herzkranken.

5. Waledinsky, Einfluss der Kohlensäurebäder auf das Elektrocardiogramm. '/eitschrift f. physikal, u. diätet. Ther. Bd. 17. H. 1.

6. Strubell, Die klinische, prognostische und therapeutische Bedeutung des Elektrocardiogramms. Med. Klinik. 1912. Nr. 30 u. „Das Wechselstrombad ", Dresden u. Leipzig 1913. Verlag von Theodor Steinkopff.

7. H. Jastrowitz, Versuche über die Beeinflussung des Elektrocardiogramms durch hydriatische Proceduren. Diese Zoitschr. 1913. Bd. 14.

8. Theo Grödel, Koblensäurebäder bei atrioventrikulärem Block. Münchner med. Wochenschr. 1912. Nr. 14.

9. Kellermann, Ueber die Beeinflussung des leitungswiderstandes des menschlichen Körpers für den galvanischen Strom durch bydrotherapeutische Proceduren. Diese Zeitschr. 1905. Bd 2 . 